\title{
Density Functional Theory with Modified Dispersion Correction for Metals Applied to Self-Assembled Monolayers of Thiols on $\mathrm{Au}(111)$
}

\author{
M. P. Andersson \\ Nano-Science Center, Department of Chemistry, University of Copenhagen, Universitetsparken 5, 2100 Copenhagen, Denmark \\ Correspondence should be addressed to M. P. Andersson; ma@nano.ku.dk
}

Received 12 June 2013; Accepted 22 August 2013

Academic Editors: Q. Ge, A. Kokalj, and M. Koyama

Copyright (C) 2013 M. P. Andersson. This is an open access article distributed under the Creative Commons Attribution License, which permits unrestricted use, distribution, and reproduction in any medium, provided the original work is properly cited.

\begin{abstract}
Using sound physical principles we modify the DFT-D2 atom pairwise semiempirical dispersion correction to density functional theory to work for metallic systems and in particular self-assembled monolayers of thiols on gold surfaces. We test our approximation for two functionals PBE-D and revPBE-D for lattice parameters and cohesive energies for $\mathrm{Ni}, \mathrm{Pd}, \mathrm{Pt}, \mathrm{Cu}, \mathrm{Ag}$, and $\mathrm{Au}$, adsorption energies of $\mathrm{CO}$ on (111) surfaces of $\mathrm{Pd}, \mathrm{Pt}, \mathrm{Cu}, \mathrm{Ag}$, and $\mathrm{Au}$, and adsorption energy of benzene on $\mathrm{Ag}(111)$ and $\mathrm{Au}(111)$. Agreement with experimental data is substantially improved. We apply the method to self-assembled monolayers of alkanethiols on $\mathrm{Au}(111)$ and find reasonable agreement for PBE-D and revPBE-D for both physisorption of n-alkanethiols as well as dissociative chemisorption of dimethyl disulfide as an Au-adatom-dithiolate complex. By modifying the $\mathrm{C}_{6}$ coefficient for $\mathrm{Au}$, we obtain quantitative agreement for physisorption and chemisorption for both PBE-D and revPBE-D using the same set of parameters. Our results confirm that inclusion of dispersion forces is crucial for any quantitative analysis of the thiol and thiolate bonds to the gold surface using quantum chemical calculations.
\end{abstract}

\section{Introduction}

Density functional theory (DFT) is the method of choice for first-principles calculations in condensed matter systems and has contributed greatly to our understanding of metallic systems such as heterogeneous catalysis of ammonia synthesis [1].

However, conventional DFT functionals do not take into account van der Waals interactions, that is, London dispersion. These interactions are crucial for many systems such as interlayer bonding in graphite [2] and biological systems. Research in the last decade has led to dispersion being included in DFT in many ways [3]. Some methods that have the correct asymptotic $1 / r^{6}$ behavior are nonlocal dispersiondensity functional (vdW-DF) $[4,5]$ and semiempirical atom pairwise dispersion [6-9]. Some highly parameterized metaGGA functionals also include short-range dispersion effects, like the M06 family of functionals [10], but do not have the correct long-range asymptotic behavior [3]. The vdWDF functional takes into account electronic effects such as electron transfer from first-principles. Its accuracy for normal thermochemistry is however not well established yet. It is furthermore not well defined for spin-polarized systems, such as $\mathrm{Fe}, \mathrm{Ni}, \mathrm{Co}$, and their alloys.

Dispersion effects included via semiempirical atom pairwise interactions using the DFT-D2 or DFT-D3 methods by Grimme et al. have been shown to give quite accurate thermochemistry for both covalently bonded systems and systems dominated by dispersion forces [9]. They also have advantages in that they have negligible computational time and add valuable insight, because the contribution from dispersion is easily separated out from the standard DFT energy. Another advantage is that when using DFT-D, the difference between various DFT functionals tends to decrease [9], as the global scaling factor $s_{6}$ is larger for more local functionals and smaller for functionals with stronger longrange interactions. For PBE $s_{6}=0.75$, and for revPBE $s_{6}=1.25$. Semiempirical atom pairwise dispersion interactions have been shown to work well for both bulk and surface properties of nonmetallic condensed systems, with 
the original parameters, for example, see $[2,11-14]$, and for parameters specifically modified for ionic surfaces [15]. The DFT-D3 method is a refinement of the DFT-D2 and has been implemented and evaluated in a plane-wave code recently [16]. The authors found that physisorption on $\mathrm{Ag}(111)$ was too strong, but this could be alleviated through inclusion of threebody terms. Other studies of adsorption on metal surfaces using dispersion corrected DFT have found that adsorption energies are overestimated [17], unless $C_{6}$ parameters are decreased by fitting to ab initio data for metal clusters [18] or by including screening effects $[19,20]$.

vdW-DF has been used as a nonlocal correction to adsorption for a few metallic systems. Examples include benzene on noble metals [21], benzene and carbon monoxide on Au stepped surfaces [22], azobenzene on $\operatorname{Ag}(111)$ [23], and carbon monoxide on $\mathrm{Pt}(111)$ [24].

The focus of our paper is self-assembled monolayers (SAMs) of alkanethiols on gold [25]. Experimental data show that gold adatoms are part of the bond between the SAM and the gold surface [26-30]. Rather subtle changes to the thiol chain length and chemistry can also change the observed structure of the SAM [26]. Pure DFT for thiol chemistry on gold is far from being able to quantitatively reproduce the adsorption behavior [31], and results depend strongly on the chosen functional [32]. This could in fact primarily be a result of neglecting the dispersion contribution. Dispersion forces determine interchain interactions, and it is reasonable to assume that dispersion interaction is important for interactions between sulphur and large metal atoms such as gold (the $C_{6}$ coefficient for $\mathrm{S}$ is more than three times larger than that for $\mathrm{C}$ ). The overly attractive PBE functional includes dispersion to a small degree and predicts weak binding in noble gas dimers, where more repulsive functionals like BLYP or revPBE do not bind at all [3].

In this paper we explain the main reasons why semiempirical dispersion treatment based on atom pairwise potentials is inadequate for metallic systems and propose a simple method for how to build a physically sound yet simple model for including dispersion forces for metals based on the DFT$\mathrm{D}$ method. The method is not as rigorous as the explicit method in [19] but captures many of the same properties and has the advantage that it can be used together with the common method DFT-D2, which is available in most planewave codes. We apply it to physisorption of some linear alkanethiols as well as chemisorption of methylthiolate on $\mathrm{Au}(111)$ and show that dispersion forces account for about half of the adsorption energy of methylthiolate.

\section{Computational Details}

We start by noting that in the DFT-D atom pairwise method no screening of the dispersion interaction is taken into account. Most of the net dispersion energy comes from frequencies of the polarizability that lie in the visible part of the electromagnetic spectrum (Figure 6.3 [38]). The plasma frequency of metals is also in the visible region [39], and therefore metals will quite efficiently screen dispersion interactions, making them even more short-ranged than non-metallic systems. Although this screening depends on the whole spectrum of dynamic polarizabilities in the system, we simplify the general behavior into a model that is much easier to implement in any plane-wave code with the DFT-D2 method available. We treat the valence electrons as being inactive for dispersion interactions and assume that only the bound core electrons contribute to the atom pairwise interaction in DFT-D. The valence electrons constitute the metallic states and will screen the dispersion interaction between the ionic cores. We introduce a hard cutoff for London dispersion of 12 bohr to simulate the screening of the valence electrons. We also replace atomic $C_{6}$ coefficients and dispersion radii for any transition metal by the noble gas in the row above that metal in the periodic table. Because the DFT-D2 method only includes $C_{6}$ parameters, no higher terms in the dispersion expansion have been considered. This might be necessary for similar treatment of the DFT-D3 method. The screening distance is chosen, rather arbitrarily, as the distance where the calculated lattice parameters for PBE-D and revPBE-D agree for gold, with the goal in mind to minimize differences between density functionals. The screening represented in our work by a hard cutoff has been included more rigorously using the Lifshitz-Zaremba-Kohn (LZK) theory for dispersion interaction between an atom and a solid surface [19]. The many-body effect of the metallic screening can be rewritten in terms of pairwise potentials with reduced atomic $C_{6}$ parameters. This suggests that our simple alternative approach for a pairwise method is reasonable. Our approach treats all transition metals in the same row equally, while the LZK theory shows that the screening varies slightly depending on the metal.

Our method includes the major physics, makes use of existing implementations of current DFT packages, and provides a quick set of consistent parameters for all transition metals for use as is or as a starting point for further parameter refinement. Although in principle a hard cutoff makes the potential energy surface discontinuous, no problems were encountered during any geometry optimizations in this work. In a few occasions the BFGS optimization procedure failed, but using damped dynamics worked for all cases. Because of the cutoff introduced to handle screening from metallic states any long-range asymptotic interactions are missing for the parts of the simulation cell, which have no metal atoms, such as interchain interactions in SAMs. Both of these effects could be improved by making the cutoff via a smooth function that also depends on the position in the cell (i.e., is only active inside the metal).

\section{DFT Calculations}

All DFT calculations were performed using plane waves and ultrasoft pseudopotentials using Quantum Espresso (QE) version 4.2.1 [41]. The kinetic energy cutoff was $25 \mathrm{Ry}$ and the density cutoff 250 Ry for all adsorption studies and 35/350 Ry for bulk calculations. Adsorption energies were converged to within $0.02 \mathrm{eV}$ using these parameters. All pseudopotentials were taken from the QE pseudopotential library [42]. Both the PBE [43] and revPBE [44] functionals have been used 


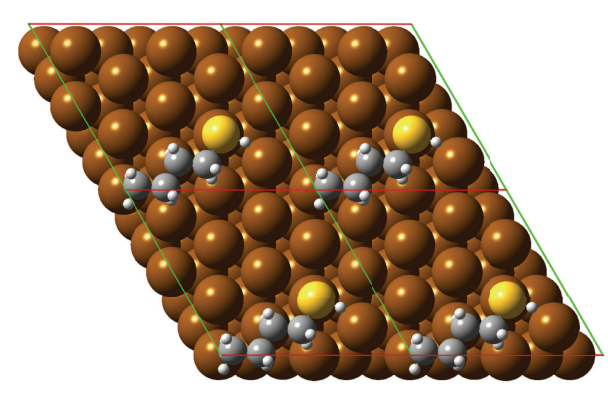

(a)

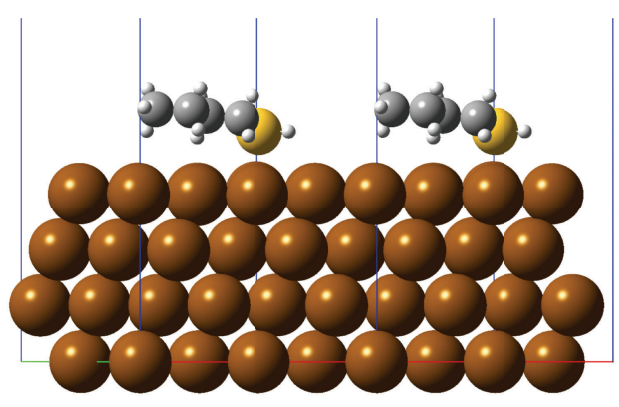

(b)

Figure 1: Molecular model of butanethiol physisorbed on $\mathrm{Au}(111)$ in top view (a) and side view (b). Color code: Au: orange, S: yellow, C: grey, and $\mathrm{H}$ : white.

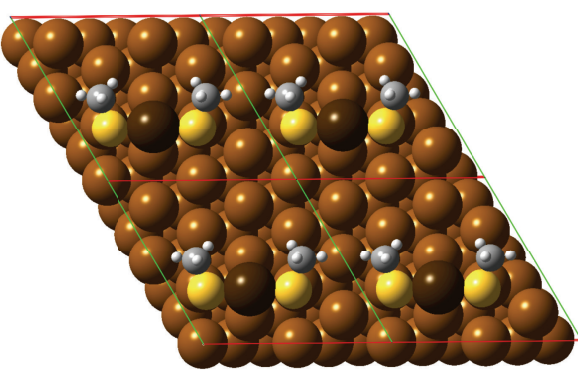

(a)

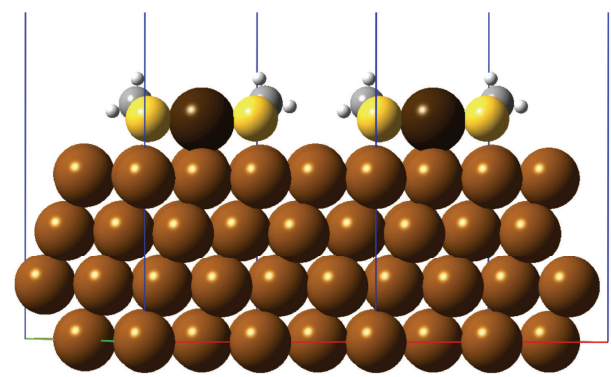

(b)

Figure 2: Optimized geometry of dissociated dimethyl disulfide on $\mathrm{Au}(111)$ adsorbed in a dithiolate-Au adatom surface complex in top view (a) and side view (b). Color code: Au: orange, Au adatom: brown, S: yellow, C: grey, and H: white.

in the paper. All surface slab calculations are made at the equilibrium lattice parameter for the corresponding functional and included four metal layers and at least $10 \AA$ vacuum between the slabs. A Gaussian smearing of 0.01 Ry was used for the Brillouin zone integration.

The modules/mm_dispersion. 990 file of $\mathrm{QE}$ was modified before the program was compiled: the $C_{6}$ coefficients and dispersion radii were taken from the DFT-D2 data in the dftd3.f file downloadable from the DFT-D3 web page [45]. The parameters for all transition metals were replaced by the noble gas in the row above. We also had to increase the parameter $m \times r$ by a factor of 16 to handle the short cutoff values we use for London dispersion. The modified mm_dispersion.f90 files are available upon request from the author.

Bulk calculations were made for the six metals $\mathrm{Cu}, \mathrm{Ag}$, $\mathrm{Au}, \mathrm{Ni}, \mathrm{Pd}$, and $\mathrm{Pt}$, and we calculated lattice parameters and cohesive energies using a $6 \times 6 \times 6$ Monkhorst Pack k-point mesh [46]. Calculations for Ni were spin polarized.

We have studied CO adsorption on the close packed (111) surfaces of the fcc metals $\mathrm{Cu}, \mathrm{Ag}, \mathrm{Au}, \mathrm{Pd}$, and Pt. The choice of metals covers the range from very weak adsorption to strong chemisorption and experimentally different adsorption sites. The CO coverage was 0.25 monolayers, and a $2 \times 2$ surface unit cell was used. The Monkhorst-Pack grid for k-points was $4 \times 4 \times 1$. All $\mathrm{CO}$ adsorption energies have been corrected using an empirical method based on the vibrational frequency related to the surface coordination [33, 47]. The correction to the adsorption energy is $1.8-0.0008 * v_{\mathrm{CO}}$, where $v_{\mathrm{CO}}$ is the internal stretch frequency of the adsorbed $\mathrm{CO}$ molecule. We used the vibrational frequencies from [33]. The adsorption energy is as a result corrected by (on average) $+0.11 \mathrm{eV}$ for top adsorption, $+0.25 \mathrm{eV}$ for bridge adsorption, and $+0.32 \mathrm{eV}$ for hollow adsorption.

Benzene adsorption on $\operatorname{Ag}(111)$ and $\mathrm{Au}(111)$ serves as an example of physisorbed systems for which dispersion is the main mode of binding and adsorption on $\mathrm{Pt}(111)$ as a system where benzene adsorbs quite strongly. These calculations were performed with a $4 \times 4$ unit cell and a $2 \times 2 \times 1$ Monkhorst Pack k-point mesh.

For alkanethiol adsorption on $\mathrm{Au}(111)$ we have used a $2 \sqrt{3} \times 2 \sqrt{3} R 30^{\circ}$ unit cell, with a $2 \times 2 \times 1$ Monkhorst Pack kpoint mesh [46]. This cell allows for physisorption of up to $C_{4}$ thiols without strong interchain interactions. The optimized butanethiol adsorption geometry is shown in Figure 1.

We have also studied methylthiolate adsorption on $\mathrm{Au}(111)$ in several adsorption geometries: on the (111) surface, adsorbed on top of an Au adatom on (111), and as a dithiol-Au adatom complex on (111). All adsorption energies presented for adatom systems include the formation energy of the adatom from a reservoir of bulk gold atoms [31]. The most stable adsorption geometry is the cis conformation of the dithiol-Au-adatom complex, consistent with a previous study [31]. The complex is shown in Figure 2. 
TABLE 1: (a) Errors in equilibrium lattice parameters for some transition metals. (b) Errors in cohesive energies for some transition metals.

(a)

\begin{tabular}{|c|c|c|c|c|c|c|c|}
\hline Metal & $\begin{array}{c}\text { Experiment } \\
(\AA)\end{array}$ & $\begin{array}{l}\text { calc - exp } \\
\text { PBE (\%) }\end{array}$ & $\begin{array}{c}\text { calc }-\exp \\
\text { PBE-D } \\
\text { (unmodified } \\
\text { DFT-D2) (\%) }\end{array}$ & $\begin{array}{l}\text { calc - exp } \\
\text { PBE-D (\%) }\end{array}$ & $\begin{array}{l}\text { calc - exp } \\
\text { revPBE (\%) }\end{array}$ & $\begin{array}{l}\text { calc }- \text { exp } \\
\text { revPBE-D } \\
\text { (unmodified } \\
\text { DFT-D2) (\%) }\end{array}$ & $\begin{array}{c}\text { calc - exp } \\
\text { revPBE-D (\%) }\end{array}$ \\
\hline $\mathrm{Ni}$ & 3.52 & -0.1 & -1.8 & -0.7 & 0.7 & -2.2 & -0.3 \\
\hline $\mathrm{Cu}$ & 3.61 & 1.7 & 0.0 & 1.1 & 2.7 & -0.3 & 1.8 \\
\hline $\mathrm{Pd}$ & 3.89 & 2.3 & 1.1 & 1.7 & 3.0 & 0.9 & 2.0 \\
\hline $\mathrm{Ag}$ & 4.09 & 1.8 & 1.5 & 1.4 & 2.6 & 2.8 & 2.0 \\
\hline $\mathrm{Pt}$ & 3.92 & 2.1 & -1.3 & 0.8 & 2.6 & -3.0 & 1.2 \\
\hline $\mathrm{Au}$ & 4.08 & 2.5 & -1.4 & 1.5 & 3.2 & -3.5 & 1.6 \\
\hline $\mathrm{MD}$ & & 1.7 & -0.3 & 1.0 & 2.5 & -0.9 & 1.4 \\
\hline MAD & & 1.8 & 1.2 & 1.2 & 2.5 & 2.1 & 1.5 \\
\hline
\end{tabular}

MD: mean deviation; MAD: mean absolute deviation.

(b)

\begin{tabular}{|c|c|c|c|c|c|c|c|}
\hline Metal & $\begin{array}{c}\text { Experiment } \\
(\mathrm{eV})\end{array}$ & $\begin{array}{l}\text { calc - exp } \\
\text { PBE }(\%)\end{array}$ & $\begin{array}{c}\text { calc - exp } \\
\text { PBE-D } \\
\text { (unmodified } \\
\text { DFT-D2) (\%) }\end{array}$ & $\begin{array}{c}\text { calc - exp } \\
\text { PBE-D (\%) }\end{array}$ & $\begin{array}{c}\text { calc - exp } \\
\operatorname{revPBE}(\%)\end{array}$ & $\begin{array}{c}\text { calc - exp } \\
\text { revPBE-D } \\
\text { (unmodified } \\
\text { DFT-D2) (\%) }\end{array}$ & $\begin{array}{c}\text { calc }-\exp \\
\text { revPBE-D (\%) }\end{array}$ \\
\hline $\mathrm{Ni}$ & 4.44 & 7.2 & 17.9 & 10.8 & -3.3 & 14.4 & 2.6 \\
\hline $\mathrm{Cu}$ & 3.49 & -5.2 & 6.2 & -1.3 & -17.0 & 1.8 & -10.8 \\
\hline $\mathrm{Pd}$ & 3.89 & 15.8 & 31.5 & 21.4 & 3.5 & 29.4 & 12.6 \\
\hline $\mathrm{Ag}$ & 2.95 & -15.5 & 3.7 & -9.6 & -29.2 & 2.7 & -19.6 \\
\hline $\mathrm{Pt}$ & 5.84 & -8.5 & 20.9 & 4.3 & -17.8 & 32.9 & -5.2 \\
\hline $\mathrm{Au}$ & 3.01 & -21.8 & 16.4 & -12.8 & -34.6 & 31.2 & -19.8 \\
\hline $\mathrm{MD}$ & & -4.7 & 16.1 & 2.1 & -16.4 & 18.7 & -6.7 \\
\hline MAD & & 12.3 & 16.1 & 10.0 & 17.6 & 18.7 & 11.8 \\
\hline
\end{tabular}

MD: mean deviation; MAD: mean absolute deviation.

Adsorption energies $E_{\text {ads }}$ were calculated in a standard fashion:

$$
E_{\mathrm{ads}}=E(\text { surface }+ \text { molecule })-E(\text { surface })-E(\text { molecule }) .
$$

This means that the more negative the adsorption energy, the stronger the molecule adsorbs on the surface.

\section{Results}

4.1. Bulk Metals. The inclusion of dispersion forces in our modified DFT-D2 method improves lattice parameters and cohesive energies for both $\mathrm{PBE}$ and revPBE (Table 1). The mean deviation, mean absolute deviation, and maximum deviation of the lattice parameters are all improved, and the results for the two dispersion corrected functionals are similar. The calculated cohesive energies behave similarly. This indicates that bulk properties of the late transition metals improve using the dispersion correction, with no major shortcomings. Furthermore, the difference between the revPBE and $\mathrm{PBE}$ functionals decreases significantly when dispersion is included. Unmodified DFT-D2 improves lattice constants and worsens cohesive energies compared to nondispersion corrected functionals, while our modified version improves both lattice parameters and cohesive energies.

4.2. Chemisorption of $\mathrm{CO}$ and Physisorption of Benzene. Adsorption energies for $\mathrm{CO}$ on transition metals are significantly improved for revPBE-D as compared to revPBE, whereas results on average are unchanged for PBE-D as compared to PBE (Table 2(a)). PBE gives stronger chemisorption than revPBE, consistent with a previous comparison between similar functionals PW91 and RPBE [33]. Weak adsorption is improved for PBE-D whereas strong chemisorption is overestimated. Again the difference between the two functionals is greatly reduced. The dispersion correction does not change the predicted adsorption site on either Pd or $\mathrm{Pt}$, where multiple sites were investigated. We performed a calculation for CO on Pt(111) using the vdW-DF2 functional as well, which gives an adsorption energy of $+0.23 \mathrm{eV}$, clearly different from $\mathrm{PBE}$ and revPBE calculations and very far from experiments. The vdW-DF was successful in solving the $\mathrm{CO} / \mathrm{Pt}(111)$ puzzle when used as a postprocessing tool [24], 
TABLE 2: (a) Adsorption energies of carbon monoxide on some late fcc transition metal (111) surfaces. All energies are in eV and include an empirical correction factor [33]. The PBE and revPBE results are calculated from the corresponding PBE-D and revPBE-D results with the dispersion contribution removed. (b) Adsorption energy of benzene on $\mathrm{Ag}(111), \mathrm{Au}(111)$, and $\mathrm{Pt}(111)$. All energies are in eV. The PBE and revPBE results are calculated from the corresponding $\mathrm{PBE}-\mathrm{D}$ and revPBE-D results with the dispersion contribution removed.

(a)

\begin{tabular}{|c|c|c|c|c|c|}
\hline Metal-adsorption site & Experiment & PBE-D & revPBE-D & $\mathrm{PBE}$ & revPBE \\
\hline $\mathrm{Cu}(111)$-top & -0.50 & -0.74 & -0.59 & -0.56 & -0.31 \\
\hline $\operatorname{Ag}(111)$-top & -0.28 & -0.19 & -0.06 & -0.04 & 0.17 \\
\hline $\mathrm{Au}(111)$-top & -0.40 & -0.28 & -0.16 & -0.03 & 0.28 \\
\hline $\mathrm{Pd}(111)$-top & & -1.36 & -1.22 & -1.16 & -0.90 \\
\hline Pd(111)_fcc hollow & -1.48 & -1.78 & -1.56 & -1.60 & -1.25 \\
\hline $\mathrm{Pt}(111)$ - top & -1.37 & -1.64 & -1.51 & -1.43 & -1.15 \\
\hline Pt(111)_fcc hollow & & -1.63 & -1.45 & -1.40 & -1.06 \\
\hline $\mathrm{MD}$ & & -0.12 & 0.03 & 0.08 & 0.35 \\
\hline MAD & & 0.20 & 0.15 & 0.17 & 0.35 \\
\hline
\end{tabular}

MD: mean deviation; MAD: mean absolute deviation.

(b)

\begin{tabular}{lcccrr}
\hline Metal surface & Experiment & PBE-D & revPBE-D & PBE & 0.05 \\
$\mathrm{Ag}(111)$ & $-0.42^{\mathrm{a}}$ & -0.50 & -0.52 & -0.88 & 0.41 \\
$\mathrm{Au}(111)$ & $-0.62^{\mathrm{b}}$ & -0.73 & -1.69 & -01 & 0.47 \\
$\mathrm{Pt}(111)$ & $-1.70^{\mathrm{c}}$ & -1.82 & & -0.81 & 0.03 \\
\hline
\end{tabular}

${ }^{a} \operatorname{Ref}[34,35]$.

${ }^{\mathrm{b}} \operatorname{Ref}[36]$.

${ }^{c} \operatorname{Ref}[37$ ]. Depends on coverage: -1.70 is at coverage 0.4 , corresponding to our setup.

whereas our results indicate that a self-consistent calculation gives quite different results.

We reoptimized the geometries for adsorption of $\mathrm{CO}$ on $\mathrm{Pt}(111)$ with PBE (no dispersion), which gave adsorption energies $-1.43 \mathrm{eV}$ (top) and $-1.42 \mathrm{eV}$ (hollow). This shows that the adsorption geometries for chemisorbed $\mathrm{CO}$ are not changed significantly from the PBE-D geometries, in particular considering that $\mathrm{Pt}$ has a strong dispersion interaction. We also performed a set of calculations using PBE$\mathrm{D}$ (unmodified Grimme) and found adsorption energies of $-1.92 \mathrm{eV}$ (top) and $-1.84 \mathrm{eV}$ (hollow). The unmodified dispersion correction thus strongly overbinds this system and is less suitable for adsorption onto metal surfaces if used as is.

For adsorption of benzene on $\mathrm{Ag}(111)$ both functionals give good results, overestimating the experimental adsorption energy $(-0.42 \mathrm{eV}[34,35])$ by about $0.1 \mathrm{eV}$ (Table 2(b)). $\mathrm{On} \mathrm{Au}(111)$, the error is slightly larger; PBE-D overestimates the experimental adsorption energy $(-0.62 \mathrm{eV}[36])$ by $0.13 \mathrm{eV}$ and revPBE-D by $0.28 \mathrm{eV}$. As expected, the entire binding energy is a result of dispersion. The calculations for strongly bound benzene on $\mathrm{Pt}(111)$ show that both functionals match the experimental adsorption energy $-1.70 \mathrm{eV}$ very well (Table 2(b)). Our approach gives equally good or better results for benzene adsorption on $\mathrm{Au}(111)$ and $\mathrm{Ag}(111)$ than Møller-Plesset 2nd order perturbation theory (MP2) [35], at a much smaller computational cost. We have also performed calculations for benzene on $\mathrm{Au}(111)$ with a cutoff for London dispersion of $200 \mathrm{Bohr}$, and the adsorption energy is then overestimated by $\sim 0.3 \mathrm{eV}$ for PBE-D and $\sim 0.55 \mathrm{eV}$ for revPBE-D, showing the importance of keeping the dispersion forces short-ranged via the hard cutoff.

4.3. Self-Assembled Monolayers of Thiols on Au(111). We start our discussion of alkanethiols on $\mathrm{Au}(111)$ with the physisorption of methanethiol, $\mathrm{CH}_{3} \mathrm{SH}$, up to butane thiol, $\mathrm{CH}_{3}\left(\mathrm{CH}_{2}\right)_{3} \mathrm{SH}$. The experimental physisorption energy is linearly dependent on the chain length for nonbranched thiols [40], and the slope of the line is thus related to the strength of the $\mathrm{CH}_{2}-\mathrm{Au}$ interaction, whereas the $y$-intercept is related to the $\mathrm{SH}-\mathrm{Au}$ interaction [40]. Our calculated physisorption energies for the thiols on gold are too strong (Figure 3(a) and Table 3(a)), as was physisorption of benzene on gold. Again revPBE-D overestimates more than PBE-D. Both the slope and the $y$-intercept deviate from experiment, which indicates that both $\mathrm{Au}-\mathrm{C}$ and $\mathrm{Au}-\mathrm{S}$ dispersion interactions are too strong. No adatom geometries were investigated in this paper for physisorbed thiols.

We have also calculated the dissociative adsorption energy of dimethyl disulfide adsorbed on $\mathrm{Au}(111)$ as RS-, RS-Au-adatom and as RS-Au-SR adatom-dithiol moiety (Table 3(b)). The RS-Au-SR has previously been found to be the most stable adsorption geometry by DFT calculations [31], and the dissociative chemisorption energy of disulfides is independent of chain length [40], which results in a straight line for chemisorption in Figure 3. We also find the cis-RSAu-RS adsorption geometry to be the most stable one. 
TABLE 3: (a) Physisorption energies of ethanethiol and butanethiol on $\mathrm{Au}(111)$. All energies are in $\mathrm{eV}$. The physisorbed R-SH species are referenced against corresponding gas phase thiol. $C_{6}$ coefficients are in $\mathrm{Ry} * \mathrm{Bohr}^{6}$. (b) Dissociative adsorption energy of dimethyl disulfide as a dithiol-adatom surface complex on $\mathrm{Au}(111)$ including the formation of adatom species from bulk $\mathrm{Au}$ atoms (Figure 2). The $\mathrm{PBE}$ and revPBE results are obtained from the PBE-D and revPBE$D$ results by subtracting the contribution from dispersion forces. $C_{6}$ coefficients are in $\mathrm{Ry} * \mathrm{Bohr}^{6}$.

(a)

\begin{tabular}{|c|c|c|}
\hline Adsorbate & $\mathrm{CH}_{3} \mathrm{CH}_{2} \mathrm{SH}$ & $\mathrm{CH}_{3}\left(\mathrm{CH}_{2}\right)_{3} \mathrm{SH}$ \\
\hline Experiment $^{\mathrm{a}}$ & -0.59 & -0.70 \\
\hline PBE-D $C_{6}(\mathrm{Au})=1040$ & -0.82 & -1.00 \\
\hline $\operatorname{revPBE}-\mathrm{D} C_{6}(\mathrm{Au})=1040$ & -0.81 & -1.04 \\
\hline PBE & -0.31 & -0.31 \\
\hline revPBE & 0.20 & 0.31 \\
\hline PBE-D $C_{6}(\mathrm{Au})=520$ & -0.68 & -0.80 \\
\hline revPBE-D $C_{6}(\mathrm{Au})=520$ & -0.56 & -0.69 \\
\hline
\end{tabular}

${ }^{a}$ Experimental data are taken from [40].

(b)

\begin{tabular}{lc}
\hline Method & $\begin{array}{c}\text { Dissociative adsorption energy } \\
(\mathrm{eV})\end{array}$ \\
\hline Experiment $^{\mathrm{a}}$ & $-\mathbf{1 . 3 0}$ \\
PBE-D C $_{6}(\mathrm{Au})=1040$ & -1.67 \\
revPBE-D C $(\mathrm{Au})=1040$ & -1.65 \\
$\mathrm{PBE}$ & -0.91 \\
revPBE & -0.24 \\
PBE-D C $6(\mathrm{Au})=520$ & $-\mathbf{1 . 4 7}$ \\
revPBE-D $C_{6}(\mathrm{Au})=520$ & $-\mathbf{1 . 3 3}$ \\
\hline
\end{tabular}

${ }^{a}$ Experimental data are taken from [40].

The adsorption energy is overestimated by $0.35 \mathrm{eV}$ (Figure 3(a)), but agreement with experiments is better than for nondispersion corrected functionals [31].

Our results qualitatively reproduce TPD results of small thiol and disulfide measurements. Both physisorption and dissociative chemisorption are too strong, but the balance between the two is reasonable. If we extrapolate our results, the physisorption and the dissociative chemisorption lines cross at a chain length of 9 carbon atoms for revPBE-D and 11 carbon atoms for PBE-D. This is to be compared to experimental data for which the lines cross for linear alkanethiols with 14 carbon atoms [40].

Physisorption of benzene and thiols on $\mathrm{Au}(111)$ as well as chemisorption of thiolate on $\mathrm{Au}(111)$ are overestimated. This coupled with the fact that revPBE-D overestimates more than PBE-D implies that our simple approximation leads to an overestimation of surface-adsorbate dispersion interaction between any molecule and gold. This in turn implies that the systematic error stems from the dispersion parameters for $\mathrm{Au}$ and that the $C_{6}$ parameter is too high.

As a simple refinement and incentive to other groups, we try a $C_{6}$ coefficient for $\mathrm{Au}$ that is half as high. The adsorption energy of benzene on $\mathrm{Au}(111)$ becomes $-0.51 \mathrm{eV}$,
TABLE 4: Modified DFT-D2 parameters used for Au which give quantitative agreement for alkanethiol and alkanethiolate adsorption on $\mathrm{Au}(111)$

\begin{tabular}{lcc}
\hline Parameter & Value & Unit \\
\hline$C_{6}$ & 520 & $\mathrm{Ry} *$ Bohr $^{6}$ \\
vdW radius & 3.555 & Bohr \\
Dispersion cutoff distance & 12 & Bohr \\
\hline
\end{tabular}

which is $0.1 \mathrm{eV}$ higher than the experimental value, but on the other hand both the PBE-D and revPBE-D functionals give the same result, which is a strong indication that the $C_{6}$ coefficient is more reasonable after modification.

More importantly, the result for thiol SAM's on gold is highly encouraging (Figure 3(b)).

Both functionals now obtain the right balance between physisorption of alkanethiols and chemisorption of dialkanedithiols, with a crossing occurring for chain length $n=$ 14 where physisorption is as strong as chemisorption, in agreement with experiments. The revPBE-D results agree with experiments, while PBE-D only slightly overestimates both physisorption $(0.1 \mathrm{eV})$ and chemisorption $(0.2 \mathrm{eV})$.

Quite importantly, our results indicate that the chemisorption energy of thiols binding as a thiolate via a dithiolate$\mathrm{Au}$-adatom surface complex is strongly influenced by dispersion interactions (65\% of the total adsorption energy for revPBE-D and $40 \%$ for PBE-D using the modified DFTD2 parameters of Table 4). Probably the only reason why PBE is moderately successful for SAM calculations is because it contains some long-range effects in its construction [3], whereas revPBE does not. It also strongly suggests that any accurate theoretical treatment of thiols adsorbed on gold needs to take into account dispersion. Our parameters that do so simultaneously for PBE-D and revPBE-D for the thiol/gold system are presented in Table 4 . They should work for any molecule adsorbing onto gold surfaces.

\section{Conclusions}

We have modified the DFT-D method of Grimme [7] for use in metallic systems by introducing a hard cutoff of 12 Bohr to the dispersion interaction and replacing the $C_{6}$ coefficients for metal atoms by those of the noble gas in the row above. Our model takes into account screening of the dispersion forces by the conducting valence electrons in the metal, includes the main physics, and reproduces a wide variety of experimental data for both bulk metallic systems as well as adsorption onto metal surfaces. It is particularly useful in systems where both chemisorption and dispersion interactions compete and need to be taken into account simultaneously, such as self-assembled thiol monolayers on gold.

Our calculated adsorption energies are in agreement with experimental data for both physisorbed and dissociatively chemisorbed thiols on gold. Furthermore, our calculations show that including dispersion is crucial in order to obtain that level of accuracy for this kind of system. 


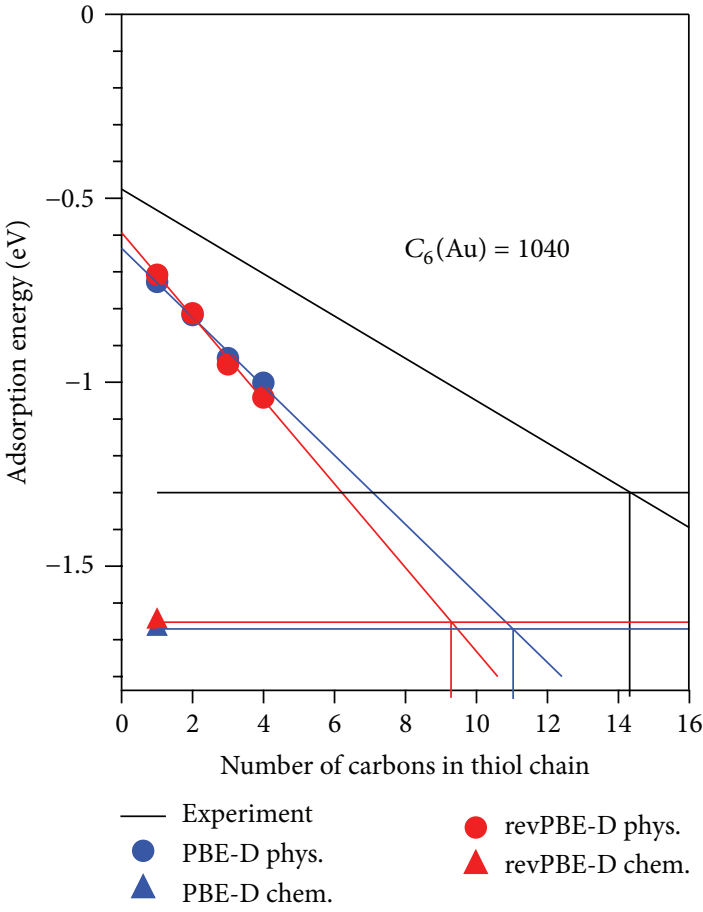

(a)

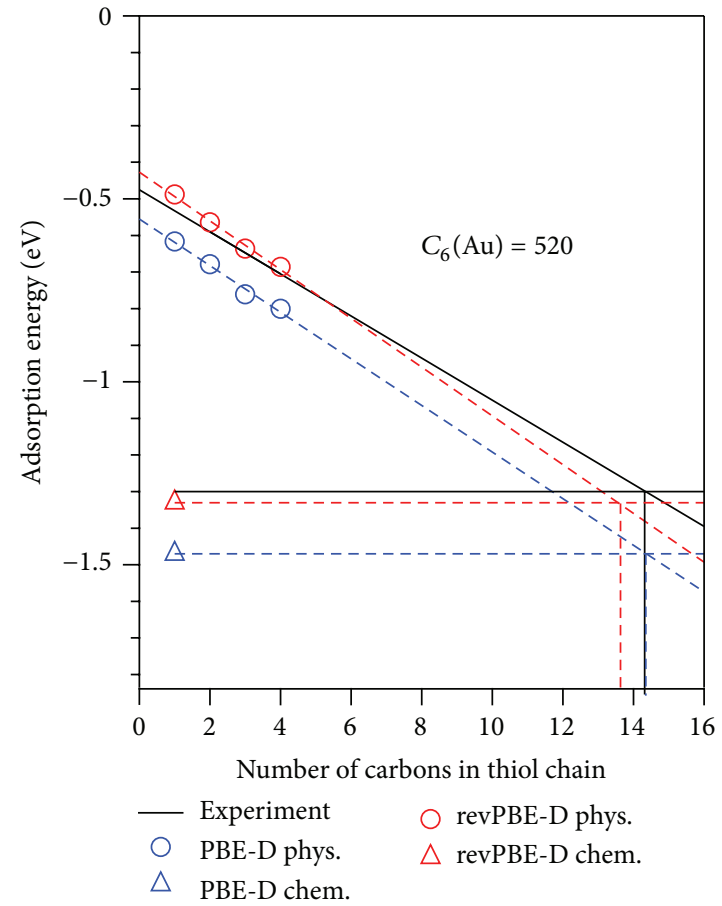

(b)

Figure 3: Comparison of experimental and calculated adsorption energies of alkanethiols (phys.) and dissociative chemisorption of dimethyl disulfide (chem.) on $\mathrm{Au}(111)$ for the modified PBE-D and revPBE-D methods. The parameters for the DFT-D method used for Au are (a) $C_{6}(\mathrm{Au})=1040 \mathrm{Ry} * \mathrm{bohr}^{6}$ and $(\mathrm{b}) C_{6}(\mathrm{Au})=520 \mathrm{Ry} * \mathrm{bohr}^{6}$.

\section{Acknowledgments}

The authors would like to thank Paolo Gianozzi and Daniel Forrer for helpful discussions regarding modifications of the mm_dispersion.f90 file in Quantum Espresso. They acknowledge financial support from Maersk Oil \& Gas A/S and BP plc. The Danish Center for Scientific Computing has provided the computing resources.

\section{References}

[1] K. Honkala, A. Hellman, I. N. Remediakis et al., "Ammonia synthesis from first-principles calculations," Science, vol. 307, no. 5709, pp. 555-558, 2005.

[2] V. Barone, M. Casarin, D. Forrer, M. Pavone, M. Sambi, and A. Vittadini, "Role and effective treatment of dispersive forces in materials: polyethylene and graphite crystals as test cases," Journal of Computational Chemistry, vol. 30, no. 6, pp. 934-939, 2009.

[3] S. Grimme, "Density functional theory with London dispersion corrections," Wiley Interdisciplinary Reviews, vol. 1, pp. 211-228, 2011.

[4] M. Dion, H. Rydberg, E. Schröder, D. C. Langreth, and B. I. Lundqvist, "Van der Waals density functional for general geometries," Physical Review Letters, vol. 92, no. 24, Article ID 246401, 2004.

[5] O. A. Vydrov and T. Van Voorhis, "Nonlocal van der Waals density functional made simple," Physical Review Letters, vol. 103, no. 6, Article ID 063004, 2009.
[6] S. Grimme, "Accurate description of van der Waals complexes by density functional theory including empirical corrections," Journal of Computational Chemistry, vol. 25, no. 12, pp. 14631473, 2004.

[7] S. Grimme, "Semiempirical GGA-type density functional constructed with a long-range dispersion correction," Journal of Computational Chemistry, vol. 27, no. 15, pp. 1787-1799, 2006.

[8] A. Tkatchenko and M. Scheffler, "Accurate molecular van der Waals interactions from ground-state electron density and freeatom reference data," Physical Review Letters, vol. 102, no. 7, Article ID 073005, 2009.

[9] S. Grimme, J. Antony, S. Ehrlich, and H. Krieg, "A consistent and accurate $a b$ initio parametrization of density functional dispersion correction (DFT-D) for the 94 elements H-Pu," Journal of Chemical Physics, vol. 132, no. 15, Article ID 154104, 2010.

[10] R. Valero, J. R. B. Gomes, D. G. Truhlar, and F. Illas, "Good performance of the M06 family of hybrid meta generalized gradient approximation density functionals on a difficult case: $\mathrm{CO}$ adsorption on $\mathrm{MgO}(001)$," Journal of Chemical Physics, vol. 129, Article ID 124710, 2008.

[11] T. S. Bučko, J. R. Hafner, S. B. Lebègue, and J. N. G. Ángyán, "Improved description of the structure of molecular and layered crystals: Ab Initio DFT calculations with van der Waals corrections," The Journal of Physical Chemistry A, vol. 114, pp. 11814-11824, 2010.

[12] G.-X. Zhang, A. Tkatchenko, J. Paier, H. Appel, and M. Scheffler, "Van der Waals interactions in ionic and semiconductor solids," Physical Review Letters, vol. 107, no. 24, Article ID 245501, 2011. 
[13] P. Tongying and Y. Tantirungrotechai, "A performance study of density functional theory with empirical dispersion corrections and spin-component scaled second-order Møller-Plesset perturbation theory on adsorbate-zeolite interactions," Journal of Molecular Structure, vol. 945, no. 1-3, pp. 85-88, 2010.

[14] M. P. Andersson and S. L. S. Stipp, "Sensitivity analysis of cluster models for calculating adsorption energies for organic molecules on mineral surfaces," Journal of Physical Chemistry C, vol. 115, no. 20, pp. 10044-10055, 2011.

[15] S. Ehrlich, J. Moellmann, W. Reckien, T. Bredow, and S. Grimme, "System-dependent dispersion coefficients for the DFT-D3 treatment of adsorption processes on ionic surfaces," ChemPhysChem, vol. 12, no. 17, pp. 3414-3420, 2011.

[16] W. Reckien, F. Janetzko, M. F. Peintinger, and T. Bredow, "Implementation of empirical dispersion corrections to density functional theory for periodic systems," Journal of Computational Chemistry, vol. 33, pp. 2023-2031, 2012.

[17] P. V. C. Medeiros, G. K. Gueorguiev, and S. Stafström, "Benzene, coronene, and circumcoronene adsorbed on gold, and a gold cluster adsorbed on graphene: structural and electronic properties," Physical Review B, vol. 85, no. 20, Article ID 205423, 2012.

[18] K. Tonigold and A. Gross, "Adsorption of small aromatic molecules on the (111) surfaces of noble metals: a density functional theory study with semiempirical corrections for dispersion effects," Journal of Chemical Physics, vol. 132, no. 22, Article ID 224701, 2010.

[19] V. G. Ruiz, W. Liu, E. Zojer, M. Scheffler, and A. Tkatchenko, "Density-functional theory with screened van der Waals interactions for the modeling of hybrid inorganic-organic systems," Physical Review Letters, vol. 108, no. 14, Article ID 146103, 2012.

[20] T. S. Chwee and M. B. Sullivan, "Adsorption studies of C6H6 on $\mathrm{Cu}$ (111), $\mathrm{Ag}$ (111), and $\mathrm{Au}$ (111) within dispersion corrected density functional theory," The Journal of Chemical Physics, vol. 137, Article ID 134703, 2012.

[21] K. Toyoda, I. Hamada, S. Yanagisawa, and Y. Morikawa, "Adsorption of benzene on noble metal surfaces studied by density functional theory with Van der Waals correction," Journal of Nanoscience and Nanotechnology, vol. 11, no. 4, pp. 2836-2843, 2011.

[22] A. K. Kelkkanen, B. I. Lundqvist, and J. K. Nørskov, "Van der Waals effect in weak adsorption affecting trends in adsorption, reactivity, and the view of substrate nobility," Physical Review B, vol. 83, no. 11, Article ID 113401, 2011.

[23] G. Li, I. Tamblyn, V. R. Cooper, H.-J. Gao, and J. B. Neaton, "Molecular adsorption on metal surfaces with van der Waals density functionals," Physical Review B, vol. 85, no. 12, Article ID 121409, 2012.

[24] P. Lazić, M. Alaei, N. Atodiresei, V. Caciuc, R. Brako, and S. Blügel, "Density functional theory with nonlocal correlation: a key to the solution of the CO adsorption puzzle," Physical Review B, vol. 81, no. 4, Article ID 045401, 2010.

[25] H. Häkkinen, "The gold-sulfur interface at the nanoscale," Nature Chemistry, vol. 4, pp. 443-455, 2012.

[26] M. Yu, N. Bovet, C. J. Satterley et al., "True nature of an archetypal self-assembly system: mobile Au-thiolate species on Au(111)," Physical Review Letters, vol. 97, no. 16, Article ID 166102, 2006.

[27] P. Maksymovych, D. C. Sorescu, and J. T. Yates, "Gold-adatommediated bonding in self-assembled short-chain alkanethiolate species on the Au(111) surface," Physical Review Letters, vol. 97, no. 14, Article ID 146103, 2006.
[28] R. Mazzarello, A. Cossaro, A. Verdini et al., "Structure of a CH3S monolayer on $\mathrm{Au}(111)$ solved by the interplay between molecular dynamics calculations and diffraction measurements," Physical Review Letters, vol. 98, no. 1, Article ID 016102, 2007.

[29] N. A. Kautz and S. A. Kandel, "Alkanethiol/Au(111) selfassembled monolayers contain gold adatoms: scanning tunneling microscopy before and after reaction with atomic hydrogen," Journal of the American Chemical Society, vol. 130, no. 22, pp. 6908-6909, 2008.

[30] O. Voznyy, J. J. Dubowski, J. T. Yates Jr., and P. Maksymovych, "The role of gold adatoms and stereochemistry in self-assembly of methylthiolate on Au(111)," Journal of the American Chemical Society, vol. 131, no. 36, pp. 12989-12993, 2009.

[31] H. Grönbeck, H. Häkkinen, and R. L. Whetten, "Gold-thiolate complexes form a unique c $(4 \times 2)$ structure on Au(111)," Journal of Physical Chemistry C, vol. 112, no. 41, pp. 15940-15942, 2008.

[32] J. Gottschalck and B. Hammer, "A density functional theory study of the adsorption of sulfur, mercapto, and methylthiolate on Au(111)," Journal of Chemical Physics, vol. 116, no. 2, pp. 784790, 2002.

[33] F. Abild-Pedersen and M. P. Andersson, "CO adsorption energies on metals with correction for high coordination adsorption sites-A Density Functional Study," Surface Science, vol. 601, no. 7, pp. 1747-1753, 2007.

[34] K. J. Gaffney, C. M. Wong, S. H. Liu, A. D. Miller, J. D. McNeill, and C. B. Harris, "Femtosecond electron dynamics at the benzene/Ag(111) interface," Chemical Physics, vol. 251, no. 13, pp. 99-110, 2000.

[35] R. Caputo, B. P. Prascher, V. Staemmler, P. S. Bagus, and C. Wöll, "Adsorption of benzene on coinage metals: a theoretical analysis using wavefunction-based methods," Journal of Physical Chemistry A, vol. 111, no. 49, pp. 12778-12784, 2007.

[36] D. Syomin, J. Kim, B. E. Koel, and G. B. Elison, "Identification of adsorbed phenyl (C6H5) groups on metal surfaces: electroninduced dissociation of benzene on Au(111)," Journal of Physical Chemistry B, vol. 105, no. 35, pp. 8387-8394, 2001.

[37] H. Ihm, H. M. Ajo, J. M. Gottfried, P. Bera, and C. T. Campbell, "Calorimetric measurement of the heat of adsorption of benzene on Pt(111)," Journal of Physical Chemistry B, vol. 108, no. 38, pp. 14627-14633, 2004.

[38] J. N. Israelachvili, Intermolecular and Surface Forces, Academic Press, 3rd edition, 2010.

[39] M. A. Ordal, R. J. Bell, J. R. W. Alexander, L. L. Long, and M. R. Querry, "Optical properties of fourteen metals in the infrared and far infrared: $\mathrm{Al}, \mathrm{Co}, \mathrm{Cu}, \mathrm{Au}, \mathrm{Fe}, \mathrm{Pb}, \mathrm{Mo}, \mathrm{Ni}, \mathrm{Pd}, \mathrm{Pt}, \mathrm{Ag}, \mathrm{Ti}$, V, and W,' Applied Optics, vol. 24, pp. 4493-4499, 1985.

[40] D. J. Lavrich, S. M. Wetterer, S. L. Bernasek, and G. Scoles, "Physisorption and chemisorption of alkanethiols and alkyl sulfides on Au(111)," Journal of Physical Chemistry B, vol. 102, no. 18, pp. 3456-3465, 1998.

[41] P. Giannozzi, S. Baroni, N. Bonini et al., "QUANTUM ESPRESSO: a modular and open-source software project for quantum simulations of materials," Journal of Physics Condensed Matter, vol. 21, no. 39, Article ID 395502, 2009.

[42] P. Gianozzi, 2012, http://www.quantum-espresso.org/?page id $=190$.

[43] J. P. Perdew, K. Burke, and M. Ernzerhof, "Generalized gradient approximation made simple," Physical Review Letters, vol. 77, no. 18, pp. 3865-3868, 1996.

[44] Y. Zhang and W. Yang, "Comment on 'Generalized Gradient Approximation Made Simple,' Physical Review Letters, vol. 80, pp. 890-890, 1998. 
[45] S. Grimme, DFT-D3, http://www.thch.uni-bonn.de/tc/index .php? section=downloads\&subsection=DFT-D3\&lang=english.

[46] H. J. Monkhorst and J. D. Pack, "Special points for Brillouinzone integrations," Physical Review B, vol. 13, no. 12, pp. 51885192, 1976.

[47] S. E. Mason, I. Grinberg, and A. M. Rappe, "First-principles extrapolation method for accurate $\mathrm{CO}$ adsorption energies on metal surfaces," Physical Review B, vol. 69, no. 16, Article ID 161401, 2004. 

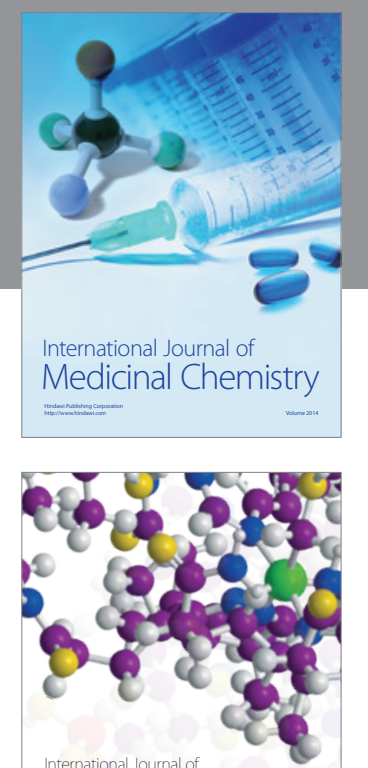

\section{Carbohydrate} Chemistry

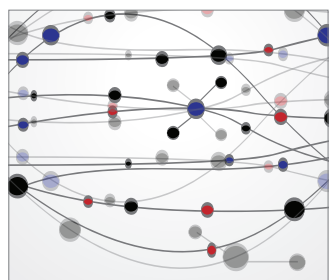

The Scientific World Journal
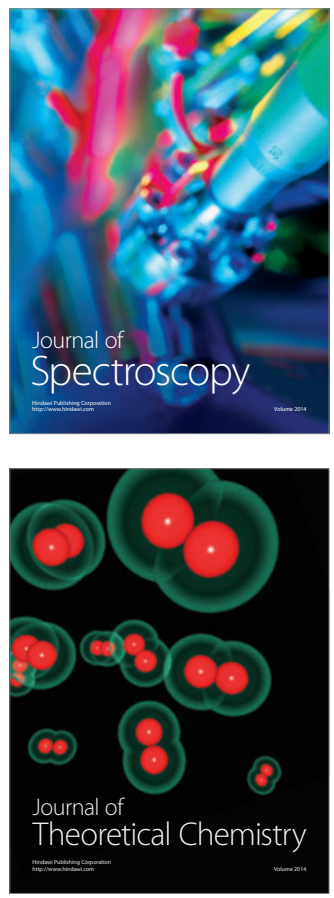
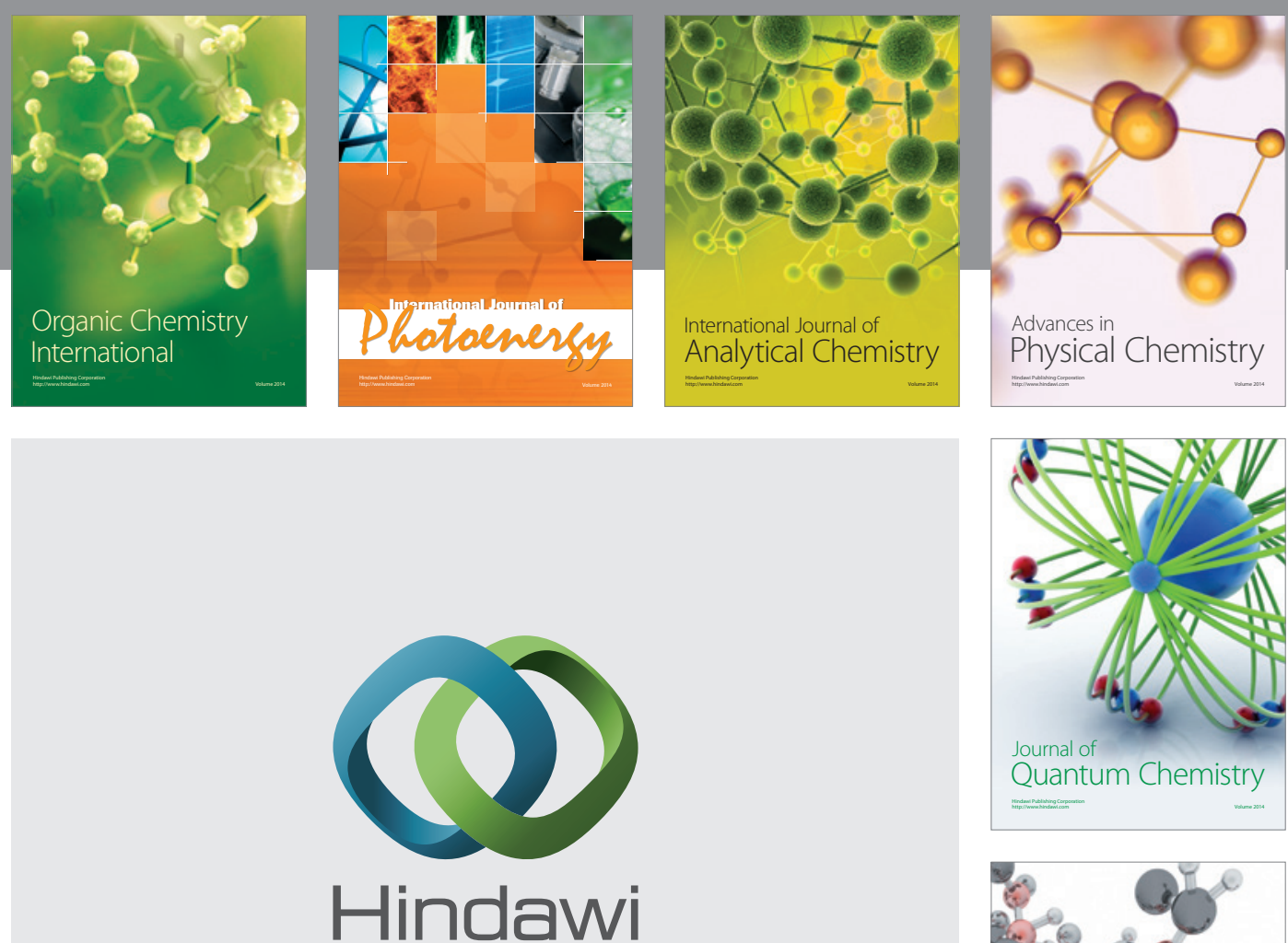

Submit your manuscripts at

http://www.hindawi.com

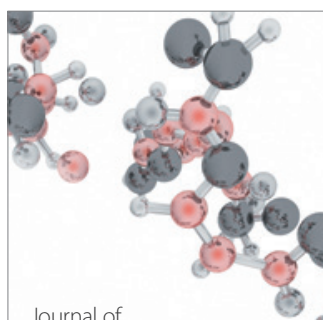

Analytical Methods

in Chemistry

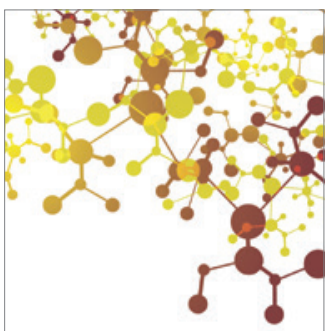

Journal of

Applied Chemistry

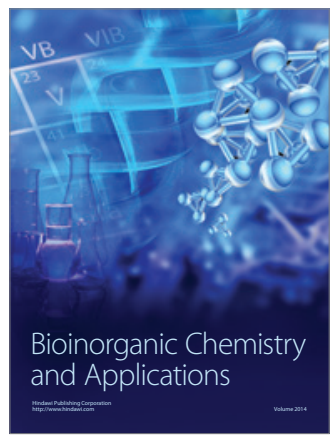

Inorganic Chemistry
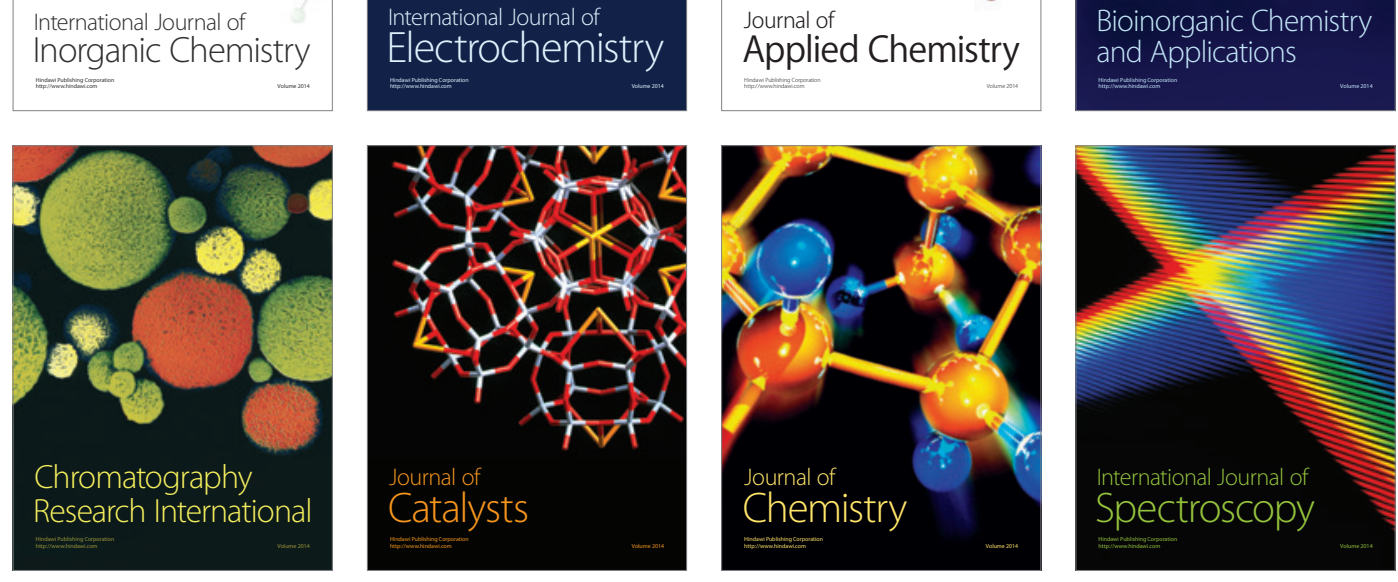